\title{
GRAND FURUTA INEQUALITY AND ITS VARIANT
}

\section{Masatoshi FuJII, EIZABURO KAMEI AND RitsuO NAKAMOTO}

Abstract. The grand Furuta inequality (GFI) is understood as follows: If positive operators $A$ and $B$ on a Hilbert space satisfy $A \geqslant B \geqslant 0, A$ is invertible and $t \in[0,1]$, then

$$
A^{1-t+r} \geqslant\left(A^{\frac{r}{2}}\left(A^{-\frac{t}{2}} B^{p} A^{-\frac{t}{2}}\right)^{s} A^{\frac{r}{2}}\right)^{\frac{1-t+r}{(p-t) s+r}}
$$

holds for $p, s \geqslant 1$ and $r \geqslant t$. In this note, we present a short proof of (GFI) which is done by the usual induction on $s$ and the use of the Furuta inequality. Furthermore we propose another simultaneous extension of the Ando-Hiai and Furuta inequalities: If $A \geqslant B \geqslant 0, A$ is invertible and $t \in[0,1]$, then

$$
A^{t} \sharp_{\frac{1-t}{p-t}} B^{p} \geqslant A^{-r+t} \sharp \frac{1-t+r}{(p-t) s+r}\left(A^{t} \bigsqcup_{s} B^{p}\right)
$$

holds for $r \geqslant t$ and $p, s \geqslant 1$. Here $\sharp \alpha$ is the $\alpha$-geometric mean and $\natural_{s}$ for $s \notin[0,1]$ is of the same form as $\sharp \alpha$.

Mathematics subject classification (2000): 47A63, 47A64.

Key words and phrases: Positive operators, operator mean, Löwner-Heinz inequality, Furuta inequality, grand Furuta inequality.

\section{REFERENCES}

[1] T. ANDO AND F. HIAI, Log majorization and complementary Golden-Thompson type inequality, Linear Alg. Appl., 197 (1994), 113-131.

[2] M. FuJII, Furuta's inequality and its mean theoretic approach, J. Operator Theory, 23 (1990), 67-72.

[3] M. FujII AND E. KAMEI, Ando-Hiai inequality and Furuta inequality, Linear Alg. Appl., 416 (2006), 541-545.

[4] M. FuJII, T. FurutA AND E. KAMEI, Furuta's inequality and its application to Ando's theorem, Linear Alg. Appl., 179 (1993), 161-169.

[5] M. FuJII, E. KAMEI AND R. NAKAMOTO, An analysis on the internal structure of the celebrated Furuta inequality, Sci. Math. Japon., 62 (2005), 421-427.

[6] T. FurutA, $A \geqslant B \geqslant 0$ assures $\left(B^{r} A^{p} B^{r}\right)^{1 / q} \geqslant B^{(p+2 r) / q}$ for $r \geqslant 0, p \geqslant 0, q \geqslant 1$ with $(1+2 r) q \geqslant p+2 r$, Proc. Amer. Math. Soc., 101 (1987), 85-88.

[7] T. FURUTA, Elementary proof of an order preserving inequality, Proc. Japan Acad., 65 (1989), 126.

[8] T. FuRUTA, Extension of the Furuta inequality and Ando-Hiai log-majorization, Linear Alg. Appl., 219 (1995), 139-155.

[9] T. FURUTA, Invitation to Linear Operators, Taylor \& Francis, London and New York, (2001).

[10] F. HIAI, Log-majorizations and norm inequalities for exponential operators, Linear Operators Banach Center Publications, vol. 38, 1997.

[11] E. KAMEI, A satellite to Furuta's inequality, Math. Japon., 33 (1988), 883-886.

[12] E. KAMEI, Parametrization of the Furuta inequality, Math. Japon., 49 (1999), 65-71.

[13] E. KAMEI, Parameterized grand Furuta inequality, Math. Japon., 50 (1999), 79-83.

[14] F. Kubo And T. Ando, Means of positive linear operators, Math. Ann., 246 (1980), 205-224. 